\title{
KANNAN TYPE MAPPING IN TVS-VALUED CONE METRIC SPACES AND THEIR APPLICATION TO URYSOHN INTEGRAL EQUATIONS
}

\author{
AKBAR AZAM AND ISMAT BEG
}

\begin{abstract}
We obtain sufficient conditions for the existence of a common fixed point of three mappings satisfying Kannan type conditions in TVS valued cone metric spaces. We also give an application by finding the solution for a system of two Urysohn integral equations. Our results generalize several well-known recent results in the literature.
\end{abstract}

\section{INTRODUCTION AND PRELIMINARIES}

A system $x=T_{i} x(i \in \Omega)$, of operator equations has one or more simultaneous solutions obtained by using the common fixed point technique. Recently Beg et al $[5,3,8,11,12]$, studied common fixed points of a pair of maps on topological vector space (TVS) valued cone metric spaces. The class of TVS cone metric spaces is larger than class of cone metric spaces, used in $[1,2,9,10,14,15,16,17]$. In this paper we obtain common fixed points and points of coincidence of three mappings in TVS-valued cone metric spaces without the assumption of normality. As an application we prove the existence of the unique solution of a system of two Urysohn integral equations. Our results improve and generalize several contemporary and recent results in the literature (e.g., see $[1,6,9,13,15,19])$.

Let $(X, d)$ be a metric space. A mapping $T: X \rightarrow X$ is called a contraction [4] if there exists $\lambda \in[0,1)$ such that

$$
d(T x, T y) \leq \lambda d(x, y),
$$

for all $x, y \in X$. Mapping $T$ is called Kannan [13] if there exists $\alpha \in\left[0, \frac{1}{2}\right)$ such that

$$
d(T x, T y) \leq \alpha[d(x, T x)+d(y, T y)]
$$

2010 Mathematics Subject Classification. 47H10, 47H09, 45N05, 46N20 54H25.

Key words and phrases. Kannan mapping, fixed point, TVS-valued cone metric space, Urysohn integral equation. 
for all $x, y \in X$. The main difference between contraction and Kannan mappings is that "contractions are always continuous where as Kannan mappings are not necessarily continuous. The Banach contraction theorem [4] is an extremely dynamic tool in mathematical analysis. However, the Kannan fixed point theorem [13] is imperative because it characterizes completeness of metric spaces [18], while Banach theorem cannot characterize the metric completeness of $X$ [7]. the Banach type contractive condition (i.e. $d(S x, T y) \leq k d(x, y))$, for a pair $S, T: X \rightarrow X$ of mappings implies that both $S$ and $T$ are equal, whereas, the condition

$$
d(S x, T y) \leq k_{1}[d(x, S x)+d(y, T y)],
$$

does not assert that $S=T$. Thus Kannan type conditions are useful to find common fixed point of a pair of nonlinear operators. An other type of contractive condition, due to Chatterjea [6] , is based on an assumption analogous to Kannan condition (2): there exists $\alpha \in\left[0, \frac{1}{2}\right)$ such that

$$
d(T x, T y) \leq \alpha[d(x, T y)+d(y, T x)]
$$

for all $x, y \in X$. It is well-known that the Banach contractions, Kannan mappings and Chatterjea mappings are independent in general.

Let $(E, \tau)$ be a topological vector space (TVS) and $P$ a subset of $E$. Then, $P$ is called a cone whenever

(i) $P$ is closed, non-empty and $P \neq\{\theta\}$,

(ii) $a x+b y \in P$ for all $x, y \in P$ and non-negative real numbers $a, b$.

(iii) $P \cap(-P)=\{\theta\}$.

For a given cone $P \subseteq E$, we can define a partial ordering $\preceq$ with respect to $P$ by $x \preceq y$ if and only if $y-x \in P$. $x \prec y$ will stand for $x \preceq y$ and $x \neq y$, while $x \ll y$ will stand for $y-x \in$ int $P$, where intP denotes the interior of $P$. A cone $\mathrm{P}$ is called solid if int $P$ is nonempty.

Definition 1. $[3,5]$ Let $X$ be a nonempty set. Suppose the mapping $d$ : $X \times X \rightarrow E$ satisfies

$\left(\mathrm{d}_{1}\right) \theta \preceq d(x, y)$ for all $x, y \in X$ and $d(x, y)=\theta$ if and only if $x=y$,

$\left(\mathrm{d}_{2}\right) d(x, y)=d(y, x)$ for all $x, y \in X$,

$\left(\mathrm{d}_{3}\right) d(x, y) \preceq d(x, z)+d(z, y)$ for all $x, y, z \in X$.

Then $d$ is called a TVS-valued cone metric on $X$ and $(X, d)$ is called a TVS-valued cone metric space.

If $E$ is a real Banach space then $(X, d)$ is called cone metric space [9].

Definition 2. [5] Let $(X, d)$ be a TVS-valued cone metric space, $x \in X$ and $\left\{x_{n}\right\}_{n \geq 1}$ a sequence in $X$. Then 
(i) $\left\{x_{n}\right\}_{n \geq 1}$ converges to $x$ whenever for every $c \in E$ with $\theta \ll c$ there is a natural number $N$ such that $d\left(x_{n}, x\right) \ll c$ for all $n \geq N$. We denote this by $\lim _{n \rightarrow \infty} x_{n}=x$ or $x_{n} \rightarrow x$.

(ii) $\left\{x_{n}\right\}_{n \geq 1}$ is a Cauchy sequence whenever for every $c \in E$ with $\theta \ll c$ there is a natural number $N$ such that $d\left(x_{n}, x_{m}\right) \ll c$ for all $n, m \geq$ $N$.

(iii) $(X, d)$ is a complete TVS valued cone metric space if every Cauchy sequence is convergent.

A pair $(F, T)$ of self-mappings on $X$ is said to be weakly compatible if $F T x=T F x$ whenever $F x=T x$. A point $y \in X$ is called point of coincidence of a family $T_{j}, j \in J$, of self-mappings on $X$ if there exists a point $x \in X$ such that $y=T_{j} x$ for all $j \in J$.

Lemma 3. [2] Let $X$ be a nonempty set and the mappings $S, T, F: X \rightarrow X$ have a unique point of coincidence $v \in X$. If $(S, F)$ and $(T, F)$ are weakly compatible, then $S, T$ and $F$ have a unique common fixed point.

\section{COMmon FIXED POINT}

Theorem 4. Let $(X, d)$ be a complete TVS-valued cone metric space, $P$ be a solid cone, and mappings $S, T, F: X \rightarrow X$ satisfy:

$$
d(S x, T y) \preceq A d(F x, S x)+B d(F y, T y),
$$

for all $x, y \in X$, where $A, B$ are non-negative real numbers with $A+B<1$. If

$$
S(X) \cup T(X) \subseteq F(X)
$$

and $F(X)$ or $S(X) \cup T(X)$ is a complete subspace of $X$, then $S, T$ and $F$ have a unique point of coincidence. Moreover if $(S, F)$ and $(T, F)$ are weakly compatible, then $S, T$ and $F$ have a unique common fixed point.

Proof. We shall first show that, if $S, T$ and $F$ have a point of coincidence, then it is unique. For this, assume that there exist two distinct points of coincidence $v, v^{*}$ of mappings $S, T$ and $F$ in $X$. It follows that there exists $u, u^{*} \in X$ such that

$$
v=F u=S u=T u,
$$

and

$$
v^{*}=F u^{*}=S u^{*}=T u^{*} .
$$

From (4), we obtain

$$
\begin{aligned}
d\left(v, v^{*}\right) & =d\left(S u, T u^{*}\right) \\
& \preceq A d(F u, S u)+B d(F u, T u) \\
& \preceq(A+B) d\left(v, v^{*}\right),
\end{aligned}
$$


it implies that

$$
v=v^{*}, \text { a contradiction. }
$$

Now, we prove the existence of a point of coincidence of the mappings $S, T$ and $F$. Let $x_{0}$ be an arbitrary point in $X$. Choose a point $x_{1}$ in $X$ such that $F x_{1}=T x_{0}$. This can be done since $S(X) \cup T(X) \subseteq F(X)$. Similarly choose a point $x_{2}$ in $\mathrm{X}$ such that $F x_{2}=S x_{1}$. Continuing this process having chosen $x_{n}$ in $X$, we obtain $x_{n+1}$ in $X$ such that

$$
\begin{aligned}
& F x_{2 n+1}=T x_{2 n} \\
& F x_{2 n+2}=S x_{2 n+1}, \quad n \geq 0 .
\end{aligned}
$$

Suppose there exists $n$ such that $F x_{2 n}=F x_{2 n+1}$. Then $F x_{2 n}=T x_{2 n}$ and from (4)

$$
\begin{aligned}
d\left(F x_{2 n}, S x_{2 n}\right) & =d\left(F x_{2 n+1}, S x_{2 n}\right) \\
& =d\left(T x_{2 n}, S x_{2 n}\right) \\
& \preceq A d\left(F x_{2 n}, S x_{2 n}\right)+B d\left(F x_{2 n}, T x_{2 n}\right) \\
& \preceq A d\left(F x_{2 n}, S x_{2 n}\right)+B d\left(F x_{2 n}, F x_{2 n+1}\right) \\
& \preceq A d\left(F x_{2 n}, S x_{2 n}\right),
\end{aligned}
$$

which yields $F x_{2 n}=S x_{2 n}$ and so, $F x_{2 n}=S x_{2 n}=T x_{2 n}=y$ (say) is the required unique point of coincidence of $F, S$ and $T$. Similarly, if $F x_{2 n+1}=$ $F x_{2 n+2}$ for some $n$. Then $F x_{2 n+1}=S x_{2 n+1}=T x_{2 n+1}=y$ is the required point. Thus in this sequel of proof we can suppose that $F x_{n} \neq F x_{n+1}$ for all $n$. From (4)

$$
\begin{aligned}
d\left(F x_{2 n}, F x_{2 n+1}\right) & =d\left(S x_{2 n-1}, T x_{2 n}\right) \\
& \preceq A d\left(F x_{2 n-1}, S x_{2 n-1}\right)+B d\left(F x_{2 n}, T x_{2 n}\right) \\
& \preceq A d\left(F x_{2 n-1}, F x_{2 n}\right)+B d\left(F x_{2 n}, F x_{2 n+1}\right) \\
& \preceq \frac{A}{1-B} d\left(F x_{2 n-1}, F x_{2 n}\right) \\
& \preceq \max \left\{\frac{B}{1-A}, \frac{A}{1-B}\right\} d\left(F x_{2 n-1}, F x_{2 n}\right),
\end{aligned}
$$

and

$$
\begin{aligned}
d\left(F x_{2 n-1}, F x_{2 n}\right) & =d\left(T x_{2 n-2}, S x_{2 n-1}\right) \\
& \preceq A\left(F x_{2 n-1}, S x_{2 n-1}\right)+B d\left(F x_{2 n-2}, T x_{2 n-2}\right) \\
& \preceq A\left(F x_{2 n-1}, F x_{2 n}\right)+B d\left(F x_{2 n-2}, F x_{2 n-1}\right) \\
& \preceq \frac{B}{1-A} d\left(F x_{2 n-2}, F x_{2 n-1}\right)
\end{aligned}
$$




$$
\preceq \max \left\{\frac{B}{1-A}, \frac{A}{1-B}\right\} d\left(F x_{2 n-2}, F x_{2 n-1}\right) .
$$

It implies that

$$
d\left(F x_{2 n}, F x_{2 n+1}\right) \preceq \lambda d\left(F x_{2 n-1}, F x_{2 n}\right),
$$

where $\lambda=\max \left\{\frac{B}{1-A}, \frac{A}{1-B}\right\}$. As $F x_{n} \neq F x_{n+1}$ and $A+B<1$, therefore $0<\lambda<1$, and for all $n$,

$$
\begin{aligned}
d\left(F x_{n}, F x_{n+1}\right) \preceq \lambda d\left(F x_{n-1}, F x_{n}\right) & \\
& \preceq \lambda^{2} d\left(F x_{n-2}, F x_{n-1}\right) \preceq \cdots \preceq \lambda^{n} d\left(F x_{0}, F x_{1}\right),
\end{aligned}
$$

Now for any $m>n$,

$$
\begin{aligned}
d\left(F x_{m}, F x_{n}\right) & \preceq d\left(F x_{n}, F x_{n+1}\right)+d\left(F x_{n+1}, F x_{n+2}\right)+\cdots+d\left(F x_{m-1}, F x_{m}\right) \\
& \preceq\left[\lambda^{n}+\lambda^{n+1}+\cdots+\lambda^{m-1}\right] d\left(F x_{0}, F x_{1}\right) \\
& \preceq\left[\frac{\lambda^{n}}{1-\lambda}\right] d\left(F x_{0}, F x_{1}\right) .
\end{aligned}
$$

Let $\theta \ll c$ be given, choose a symmetric neighborhood $V$ of $\theta$ such that $c+$ $V \subseteq \operatorname{int} P$. Also, choose a natural number $N_{1}$ such that $\left[\frac{\lambda^{n}}{1-\lambda}\right] d\left(F x_{0}, F x_{1}\right) \in$ $V$, for all $n \geq N_{1}$. Then, $\frac{\lambda^{n}}{1-\lambda} d\left(F x_{1}, F x_{0}\right) \ll c$, for all $n \geq N_{1}$. Thus,

$$
d\left(F x_{m}, F x_{n}\right) \preceq\left[\frac{\lambda^{n}}{1-\lambda}\right] d\left(F x_{0}, F x_{1}\right) \ll c,
$$

for all $m>n$. Therefore, $\left\{F x_{n}\right\}_{n \geq 1}$ is a Cauchy sequence. Since $F X$ is complete, there exist $u \in X, v \in F X$ such that $F x_{n} \rightarrow v=F u$ (this holds also if $S(X) \cup T(X)$ is complete with $v \in S(X) \cup T(X))$. Choose a natural number $N_{2}$ such that for all $n \geq N_{2}$

$$
d\left(F x_{n+1}, F x_{n}\right) \ll \frac{c(1-B)}{2 A} \text { and } d\left(F x_{n+1}, F u\right) \ll \frac{c(1-B)}{2} .
$$

Then for all $n \geq N_{2}$

$$
\begin{aligned}
d(F u, T u) & \preceq d\left(F u, F x_{2 n+2}\right)+d\left(F x_{2 n+2}, T u\right) \\
& \preceq d\left(F u, F x_{2 n+2}\right)+d\left(S x_{2 n+1}, T u\right) \\
& \preceq d\left(F u, F x_{2 n+2}\right)+A d\left(F x_{2 n+1}, F x_{2 n+2}\right)+B d(F u, T u) \\
& \preceq \frac{1}{1-B} d\left(F u, F x_{2 n+2}\right)+\frac{A}{1-B} d\left(F x_{2 n+1}, F x_{2 n+2}\right) \\
& \ll \frac{c}{2}+\frac{c}{2}=c .
\end{aligned}
$$

Thus

$$
d(F u, T u) \ll \frac{c}{m}, \text { for all } m \geq 1 .
$$


So, $\frac{c}{m}-d(F u, T u) \in P$, for all $m \geq 1$. Since $\frac{c}{m} \rightarrow \theta($ as $m \rightarrow \infty)$ and $P$ is closed, $-d(F u, S u) \in P$. But $d(F u, T u) \in P$, therefore, $d(F u, T u)=\theta$. Hence

$$
v=F u=T u
$$

and

$$
d(F u, S u)=d(T u, S u) \preceq A d(F u, S u)+B d(F u, T u)=A d(F u, S u),
$$

implies that $v$ is a unique point of coincidence of $F, S$ and $T$. If $(S, F)$ and $(T, F)$ are weakly compatible, then by Lemma $3, v$ is a unique common fixed point of $S, T$ and $F$.

Example 5. Let $X=1,2,3$ and $E$ be the set of all real valued functions on $[0,1]$ which also have continuous derivatives on $X$. Then $E$ is a vector space over $\mathbb{R}$ under the following operations:

$$
(x+y)(t)=x(t)+y(t), \quad(\alpha x)(t)=\alpha x(t),
$$

for all $x, y \in E, \alpha \in \mathbb{R}$. Let $\tau$ be the strongest vector (locally convex) topology on $E$. Then $(X, \tau)$ is a topological vector space which is not normable and is not even metrizable. Define $d: X \times X \rightarrow E$ as follows:

$$
d(x, y)(t)=\left\{\begin{array}{cc}
0 & \text { if } x=y \\
e^{\left(\ln \frac{4}{7}+t\right)} & \text { if } x \neq y \text { and } x, y \in X-\{2\} \\
e^{t} & \text { if } x \neq y \text { and } x, y \in X-\{3\} \\
e^{(t-\ln 2)} & \text { if } x \neq y \text { and } x, y \in X-\{1\}
\end{array}\right.
$$

Let $P=\{x \in E: x(t) \geq 0$ for all $t\}$. Then $(X, d)$ is a TVS-valued cone metric space. Define a mappings $F, T: X \rightarrow X$ as follows:

$$
T(x)=\left\{\begin{array}{ll}
3 & \text { if } x \neq 2 \\
1 & \text { if } x=2
\end{array}, F(x)=x\right.
$$

Note that, for all $t \in[0,1]$ and for $\alpha<\frac{1}{2}$

$$
\begin{aligned}
& d\left(T(3), T(2)(t)=d(3,1)(t)=e^{\left(\ln \frac{4}{7}+t\right),}\right. \\
& \alpha[d(F(3), T(3))(t)+d(F(2), T(2))(t)] \\
& =\alpha[d(3, T(3))(t)+d(2, T(2))(t)] \\
& =\alpha[d(2,1)(t)]=\alpha e^{t} \\
& <d(T(3), T(2))(t) .
\end{aligned}
$$

Therefore the previous relevant results on fixed points [9, 13, 15, 19] and on common fixed points [1] are not applicable to obtain fixed point of $T$ and 
common fixed point of $F$ and $T$. In order to apply Theorem 4, consider the mapping $S x=3$ for each $x \in X$. Then,

$$
d(S x, T y)(t)=\left\{\begin{array}{cc}
0 & \text { if } y \neq 2 \\
e^{\left(\ln \frac{4}{7}+t\right)} & \text { if } y=2
\end{array}\right.
$$

and for $B=\frac{4}{7}$

$$
B d(F y, T y)(t)=\frac{4}{7} e^{t} \text { if } y=2 .
$$

It follows that $F, S$ and $T$ satisfy all conditions of Theorem 4 for $A=0, B=$ $\frac{4}{7}$ and we obtain $F(3)=T(3)=S(3)=3$.

In the following we use a Chatterjea type condition to obtain point of coincidence and common fixed point of three mappings on a TVS-valued cone metric space.

Theorem 6. Let $(X, d)$ be a complete TVS-valued cone metric space, $P$ be a solid cone, and mappings $S, T, F: X \rightarrow X$ satisfy:

$$
d(S x, T y) \preceq C d(F y, S x)+D d(F x, T y),
$$

for all $x, y \in X$, where $C, D$ are non-negative real numbers with $C+D<1$. If

$$
S(X) \cup T(X) \subseteq F(X),
$$

and $F(X)$ or $S(X) \cup T(X)$ is a complete subspace of $X$, then $S, T$ and $F$ have a unique point of coincidence. Moreover if $(S, F)$ and $(T, F)$ are weakly compatible, then $S, T$ and $F$ have a unique common fixed point.

Proof. It can be easily seen that if $S, T$ and $F$ have a point of coincidence, then it is unique. Let $x_{0}$ be an arbitrary point in $X$. Choose a point $x_{1}$ in $X$ such that $F x_{1}=T x_{0}$. This can be done since $S(X) \cup T(X) \subseteq F(X)$. Similarly choose a point $x_{2}$ in $X$ such that $F x_{2}=S x_{1}$. Continuing this process having chosen $x_{n}$ in $X$, we obtain $x_{n+1}$ in $X$ such that

$$
\begin{aligned}
& F x_{2 n+1}=T x_{2 n} \\
& F x_{2 n+2}=S x_{2 n+1}, \quad n \geq 0 .
\end{aligned}
$$

Suppose there exists $n$ such that $F x_{2 n}=F x_{2 n+1}$. Then using (5), we obtain $F x_{2 n}=S x_{2 n}=T x_{2 n}=y$ (say) is the required unique point of coincidence of $F, S$ and $T$. Similarly, if $F x_{2 n+1}=F x_{2 n+2}$ for some $n$. Then $F x_{2 n+1}=$ $S x_{2 n+1}=T x_{2 n+1}=y$ is the required point. Thus in this sequel of proof we 
can suppose that $F x_{n} \neq F x_{n+1}$. From (5), we obtain

$$
\begin{aligned}
d\left(F x_{2 n}, F x_{2 n+1}\right)= & d\left(S x_{2 n-1}, T x_{2 n}\right) \\
\preceq & C d\left(F x_{2 n}, S x_{2 n-1}\right)+D d\left(F x_{2 n-1}, T x_{2 n}\right) \\
\preceq & D\left[d\left(F x_{2 n-1}, F x_{2 n}\right)+d\left(F x_{2 n}, F x_{2 n+1}\right)\right] \\
& \frac{D}{1-D} d\left(F x_{2 n-1}, F x_{2 n}\right),
\end{aligned}
$$

and

$$
\begin{aligned}
d\left(F x_{2 n-1}, F x_{2 n}\right) & =d\left(T x_{2 n-2}, S x_{2 n-1}\right) \\
& \preceq C d\left(F x_{2 n-2}, S x_{2 n-1}\right)+D d\left(F x_{2 n-1}, T x_{2 n-2}\right) \\
& \preceq C d\left(F x_{2 n-2}, F x_{2 n}\right) \\
& \preceq C\left[d\left(F x_{2 n-2}, F x_{2 n-1}\right)+d\left(F x_{2 n-1,} F x_{2 n}\right)\right] \\
& \preceq \frac{C}{1-C} d\left(F x_{2 n-2}, F x_{2 n-1}\right) .
\end{aligned}
$$

It follows that

$$
\begin{aligned}
d\left(F x_{2 n}, F x_{2 n+1}\right) & \preceq \frac{D}{1-D} d\left(F x_{2 n-1}, F x_{2 n}\right) \\
& \preceq \frac{D}{1-D} \frac{C}{1-C} d\left(F x_{2 n-2}, F x_{2 n-1}\right) \\
& \preceq\left[\frac{D}{1-D} \frac{C}{1-C}\right]^{n} d\left(F x_{o}, F x_{1}\right),
\end{aligned}
$$

and

$$
\begin{aligned}
d\left(F x_{2 n+1}, F x_{2 n+2}\right) & \preceq \frac{C}{1-C} d\left(F x_{2 n}, F x_{2 n+1}\right) \\
& \preceq \frac{C}{1-C}\left[\frac{D}{1-D} \frac{C}{1-C}\right]^{n} d\left(F x_{o}, F x_{1}\right) .
\end{aligned}
$$

Let

$$
\alpha=\frac{C}{1-C}, \quad \beta \frac{C}{1-C},
$$

then, as $F x_{n} \neq F x_{n+1}$ and $C+D<1$,

$$
0<\alpha \beta=\frac{C}{1-C} \frac{D}{1-D}=\frac{D}{1-C} \frac{C}{1-D}<1 .
$$


Now for $p<q$ we have,

$$
\begin{aligned}
& d\left(F x_{2 p+1}, F x_{2 q+1}\right) \preceq d\left(F x_{2 p+1}, F x_{2 p+2}\right)+d\left(F x_{2 p+2}, F x_{2 p+3}\right) \\
& +\cdots+d\left(F x_{2 q}, F x_{2 q+1}\right) \\
& \preceq \alpha[\alpha \beta]^{p} d\left(F x_{o}, F x_{1}\right)+[\alpha \beta]^{p+1} d\left(F x_{o}, F x_{1}\right) \\
& +\cdots+[\alpha \beta]^{q} d\left(F x_{o}, F x_{1}\right) \\
& \preceq\left[\alpha \sum_{i=p}^{q-1}(\alpha \beta)^{i}+\sum_{i=p+1}^{q}(\alpha \beta)^{i}\right] d\left(F x_{0}, F x_{1}\right) \\
& \preceq\left[\begin{array}{c}
\frac{\alpha(\alpha \beta)^{p}[1-\alpha \beta]^{q-p}}{1-\alpha \beta} \\
+\frac{(\alpha \beta)^{p+1}[1-\alpha \beta]^{q-p}}{1-\alpha \beta}
\end{array}\right] d\left(F x_{0}, F x_{1}\right) \\
& \preceq\left[\frac{\alpha(\alpha \beta)^{p}}{1-\alpha \beta}+\frac{(\alpha \beta)^{p+1}}{1-\alpha \beta}\right] d\left(F x_{0}, F x_{1}\right) \\
& \preceq(1+\beta)\left[\frac{\alpha(\alpha \beta)^{p}}{1-\alpha \beta}\right] d\left(F x_{0}, F x_{1}\right), \\
& d\left(F x_{2 p}, F x_{2 q+1}\right) \preceq(1+\alpha)\left[\frac{(\alpha \beta)^{p}}{1-\alpha \beta}\right] d\left(F x_{0}, F x_{1}\right), \\
& d\left(F x_{2 p}, F x_{2 q}\right) \preceq(1+\alpha)\left[\frac{(\alpha \beta)^{p}}{1-\alpha \beta}\right] d\left(F x_{0}, F x_{1}\right),
\end{aligned}
$$

and

$$
d\left(F x_{2 p+1}, F x_{2 q}\right) \preceq(1+\beta)\left[\frac{\alpha(\alpha \beta)^{p}}{1-\alpha \beta}\right] d\left(F x_{0}, F x_{1}\right) .
$$

Hence, for $0<n<m$

$$
d\left(F x_{n}, F x_{m}\right) \preceq\left[\frac{2(\alpha \beta)^{p}}{1-\alpha \beta}\right] d\left(F x_{0}, F x_{1}\right)
$$

where $p$ is the integer part of $\frac{n}{2}$. Let $\theta \ll c$ be given, choose a symmetric neighborhood $V$ of $\theta$ such that $c+V \subseteq \operatorname{int} P$. Since

$$
\lim _{p \rightarrow \infty}\left[\frac{2(\alpha \beta)^{p}}{1-\alpha \beta}\right] d\left(F x_{0}, F x_{1}\right)=\theta,
$$

there exists a natural number $N_{1}$ such that

$$
\left[\frac{2(\alpha \beta)^{p}}{1-\alpha \beta}\right] d\left(F x_{0}, F x_{1}\right) \in V
$$

for all $p \geq N_{1}$ and so

$$
\left[\frac{2(\alpha \beta)^{p}}{1-\alpha \beta}\right] d\left(F x_{0}, F x_{1}\right) \ll c, \text { for all } p \geq N_{1} .
$$


Consequently, for all $n, m \in \mathbb{N}$, with $2 N_{1}<n<m$, we have

$$
d\left(F x_{n}, F x_{m}\right) \ll c .
$$

Therefore, $\left\{F x_{n}\right\}_{n \geq 1}$ is a Cauchy sequence. Since $F X$ is complete, there exist $u \in X, v \in F X$ such that $F x_{n} \rightarrow v=F u$ (this hold also if $S(X) \cup T(X)$ is complete with $v \in S(X) \cup T(X))$. Choose a natural number $N_{2}$ such that for all $n \geq N_{2}$

$$
d\left(F x_{n+1}, F u\right) \ll \frac{c}{2 M},
$$

where $M=\max \left\{\frac{1+C}{1-D}, \frac{D}{1-D}\right\}$. Then for all $n \geq N_{2}$

$$
\begin{aligned}
d(F u, T u) & \preceq d\left(F u, F x_{2 n+2}\right)+d\left(F x_{2 n+2}, T u\right) \\
& \preceq d\left(F u, F x_{2 n+2}\right)+d\left(S x_{2 n+1}, T u\right) \\
& \preceq d\left(F u, F x_{2 n+2}\right)+C d\left(F u, S x_{2 n+1}\right)+D d\left(F x_{2 n+1}, T u\right) \\
& \preceq d\left(F u, F x_{2 n+2}\right)+C d\left(F u, F x_{2 n+2}\right)+D d\left(F x_{2 n+1}, T u\right) \\
& \preceq d\left(F u, F x_{2 n+2}\right)+C d\left(F u, F x_{2 n+2}\right) \\
& +D\left[d\left(F x_{2 n+1}, F u\right)+d(F u, T u)\right] \\
& \preceq\left(\frac{1+C}{1-D}\right) d\left(F u, F x_{2 n+2}\right)+\frac{D}{1-D} d\left(F x_{2 n+1}, F u\right) \\
& \preceq M d\left(F u, F x_{2 n+2}\right)+M d\left(F x_{2 n+1}, F u\right) \ll \frac{c}{2}+\frac{c}{2}=c .
\end{aligned}
$$

By a similar argument $F u=T u=S u$, which implies that $v$ is a unique point of coincidence of $F, S$ and $T$. If $(S, F)$ and $(T, F)$ are weakly compatible, then by Lemma 3, $v$ is a unique common fixed point of $S, T$ and $F$.

The following example shows that the above theorem is an improvement and a real generalization of results $[1,6,9,13,15,19]$.

Example 7. Let $(X, d)$ be the TVS-valued cone metric space of Example 5. Define a mappings $F, T: X \rightarrow X$ as follows:

$$
T(x)=\left\{\begin{array}{ll}
1 & \text { if } x \neq 2 \\
3 & \text { if } x=2 .
\end{array}, F(x)=x\right.
$$

Note that,for all $t \in[0,1]$ and for $\alpha<\frac{1}{2}$

$$
\begin{aligned}
& d\left(T(3), T(2)(t)=d(1,3)(t)=e^{\left(\ln \frac{4}{7}+t\right),}\right. \\
& \alpha[d(F(3), T(2))(t)+d(F(2), T(3))(t)] \\
& =\alpha[d(3, T(2))(t)+d(2, T(3))(t)]=\alpha e^{t} \\
& <d(T(3), T(2))(t),
\end{aligned}
$$


Therefore the previous relevant results on fixed points $[6,9,15,19]$ and on common fixed points [1] are not applicable to obtain fixed point of $T$ and common fixed point of $F$ and $T$. In order to apply the Theorem 6 , consider the mapping $S x=1$ for each $x \in X$. Then,

$$
d(S x, T y)(t)=\left\{\begin{array}{cc}
0 & \text { if } y \neq 2 \\
e^{\left(\ln \frac{4}{7}+t\right)} & \text { if } y=2
\end{array}\right.
$$

and for $D=\frac{4}{7}$

$$
\operatorname{Dd}(F x, T y)(t)=\frac{4}{7} e^{t} \text { if } y=2 .
$$

It follows that $S$ and $T$ satisfy all conditions of Theorem 6 and we obtain $F(1)=T(1)=S(1)=1$.

\section{Application}

In this section we prove an existence theorem for the common solution for two Urysohn integral equations. Let $X=C\left([a, b], \mathbb{R}^{n}\right), E$ is a topological vector space of Example 5

$$
P=\{x \in E: x(t) \geq 0 \text { for all } t \in[0,1]\},
$$

and $d: X \times X \rightarrow E$ is defined as follows:

$$
d(x, y)(t)=\left(\|x-y\|_{\infty}\right) e^{t} .
$$

It is easily seen that $(X, d)$ is a complete TVS-valued cone metric space.

Theorem 8. Consider the Urysohn integral equations

$$
\begin{aligned}
& x(t)=\int_{a}^{b} K_{1}(t, s, x(s)) d s+g(t), \\
& x(t)=\int_{a}^{b} K_{2}(t, s, x(s)) d s+h(t),
\end{aligned}
$$

where $t \in[a, b] \subset \mathbb{R}, x, g, h \in X$.

Suppose that $K_{1}, K_{2}:[a, b] \times[a, b] \times \mathbb{R}^{n} \rightarrow \mathbb{R}^{n}$ are such that $F_{x}, G_{x} \in X$ for each $x \in X$, where

$$
F_{x}(t)=\int_{a}^{b} K_{1}(t, s, x(s)) d s, \quad G_{x}(t)=\int_{a}^{b} K_{2}(t, s, x(s)) d s \text { for all } t \in[a, b] .
$$

If there exist $0<h<1$ such that for every $x, y \in X$

$$
\left(\left\|F_{x}(t)-G_{y}(t)+g(t)-h(t)\right\|_{\infty}\right) e^{t} \leq h M(x, y) e^{t},
$$

for all $x, y \in X$, where

$$
M(x, y)=\max \left\{\begin{array}{c}
\| F_{x}(t)+g(t)-x\left(t\left\|_{\infty},\right\| G_{y}(t)+h(t)-y(t) \|_{\infty},\right. \\
\| F_{x}(t)+g(t)-y\left(t\left\|_{\infty},\right\| G_{y}(t)+h(t)-x(t) \|_{\infty}\right.
\end{array}\right\} .
$$


Then the system of integral equations (6) and (7) have a unique common solution.

Proof. Define $F, S, T: X \rightarrow X$ by

$$
F(x)=x, \quad S(x)=F_{x}+g, \quad T\left((x)=G_{x}+h .\right.
$$

If

$$
M(x, y)=\| F_{x}(t)+g(t)-x\left(t \|_{\infty},\right.
$$

it is easily seen that

$$
\left(\|S-T\|_{\infty}\right) e^{t} \leq h\left(\|S(x)-x\|_{\infty}\right) e^{t}
$$

for every $x, y \in X$. By Theorem 4 if $A=h, B=0$, the Urysohn integral equations (6) and (7) have a unique common solution. If

$$
M(x, y)=\left\|G_{y}(t)+h(t)-y(t)\right\|_{\infty},
$$

then

$$
\left(\|S-T\|_{\infty}\right) e^{t} \leq h\left(\|T(y)-y\|_{\infty}\right) e^{t}
$$

for every $x, y \in X$. Again by Theorem 4 if $A=0, B=h$, the Urysohn integral equations (6) and (7) have a unique common solution. Similarly in other cases the result follows by Theorem 6 .

Acknowledgement. The authors sincerely thank the learned referee for a careful reading and thoughtful comments. The present version of the paper owes much to the precise and kind remarks of the referee.

\section{REFERENCES}

[1] M. Abbas and G. Jungck, Common fixed point results for non commuting mappings without continuity in cone metric spaces, J. Math. Anal. Appl., 341 (2008), 416-420.

[2] M. Arshad, A. Azam and P. Vetro, Some common fixed point results in cone metric spaces, Fixed Point Theory Appl., 2009 (2009), Article ID 493965,11 pages.

[3] A. Azam, I. Beg and M. Arshad, Fixed point in topological vector space valued cone metric spaces, Fixed Point Theory Appl., 2010 (2010), Article ID 604084, 9 pages.

[4] S. Banach, Sur les op'erations dans les ensembles abstraits et leur application aux 'equations int'egrales, Fundam. Mat., 3 (1922), 133-181.

[5] I. Beg, A. Azam and M. Arshad, Common fixed points for maps on topological vector space valued cone metric spaces, Int. J. Math. Math. Sci., 2009 (2009), Article ID. 560264,8 pages.

[6] S.K. Chatterjea, Fixed-point theorems, C.R. Acad. Bulgare Sci., 25 (1972), 727-730.

[7] E. H. Connell, Properties of fixed point spaces, Proc. Amer. Math. Soc., 10 (6) (1959), 974-979.

[8] M. Djordjević, D. Djorić, Z. Kadelburg, S. Radenović and D. Spasić, Fixed point results under c-distance in tvs-cone metric spaces, Fixed Point Theory Appl., 2011:29, doi: 10.1186/1687-1812-2011-29 (2011). 
[9] L.-G. Huang and X. Zhang, Cone metric spaces and fixed point theorems of contractive mappings, J. Math. Anal. Appl., 332 (2007), 1468-1476.

[10] S. Janković, Z. Kadelburg and S. Radenović, On cone metric spaces: a survey, Nonlinear Anal., 74 (2011), 2591-2601.

[11] Z. Kadelburg, S. Radenović, V. Rakočević, Topological vector spaces valued cone metric spaces and fixed point theorems, Fixed Point Theory Appl., 2010, Article ID 170253, (2010), 18 pages.

[12] Z. Kadelburg and S. Radenović, Coupled fixed point results under TVS-cone metric and W-cone-distance, Adv. Fixed Point Theory, 2 (1)(2012), 29-46.

[13] R. Kannan, Some results on fixed points II, Am. Math. Mon., 76 (4) (1969), 405-408.

[14] D. Ilić and V. Rakočević, Common fixed points for maps on cone metric space, J. Math. Anal. Appl., 341 (2008), 876-882.

[15] S. Rezapour and R. Hamlbarani, Some notes on paper "Cone metric spaces and fixed point theorems of contractive mappings", J. Math. Anal. Appl., 345 (2008) 719-724.

[16] S. Radenović, Common fixed points under contractive conditions in cone metric spaces, Comput. Math Appl., (2009) doi:10.1016/j.camwa.2009.07.035.

[17] S. Radenović and B.E. Rhoades, Fixed point theorem for two non-self mappings in cone metric spaces, Comput. Math. Appl.,57 (2009) 1701-1707.

[18] P. V. Subrahmanyam, Completeness and fixed-points, Monatsh. Math., 80 (4) (1975), 325-330.

[19] D. Wei-Shih, A note on cone metric fxed point theory and its equivalence, Nonlinear Anal., 72 (2010), 2259-2261.

(Received: May 10, 2012)

(Revised: June 12, 2012)

\author{
Akbar Azam \\ COMSATS Institute of \\ Information Technology \\ Islamabad- 44000 \\ Pakistan \\ akbarazam@yahoo.com \\ Ismat Beg \\ Lahore School of Economics \\ Lahore-53200 \\ Pakistan \\ begismat@yahoo.com
}

